\title{
Carbon Nanotubes by CVD and Applications
}

\author{
Alan Cassell, Lance Delzeit, Cattien Nguyen, Ramsey Stevens, Jie Han, \\ and M. Meyyappan
}

\begin{abstract}
Carbon nanotube (CNT) exhibits extraordinary mechanical and unique electronic properties and offers significant potential for structural, sensor, and nanoelectronics applications. An overview of CNT, growth methods, properties and applications is provided. Single-wall, and multi-wall CNTs have been grown by chemical vapor deposition. Catalyst development and optimization has been accomplished using combinatorial optimization methods. CNT has also been grown from the tips of silicon cantilevers for use in atomic force microscopy.
\end{abstract}

\section{INTRODUCTION}

Since Iijima's discovery [1] of carbon nanotubes (CNT) in 1991, research on the growth, characterization and application development has received significant attention. CNT is a cylindrical form of carbon, configurationally equivalent to a two-dimensional graphene sheet rolled into a seamless cylinder. The nanotubes can be single-walled (SWNT) or multiwalled (MWNT) with a concentric cylindrical arrangement. The separation between walls in multiwalled tubes is a constant $3.4 \AA$ which is the same as the interlayer separation in graphite. Several individual SWNTs can pack together to form nanotube ropes. The nanotubes are characterized by a chiral vector $c=n a+m b$ where $a$ and $b$ are vectors defining a unit cell in the planar graphene sheet and $\mathrm{n}$ and $\mathrm{m}$ are integers. Depending on chirality (i.e., the values of $n$ and $m$ ), CNT can be either metallic or semiconducting. If ( $n$ $\mathrm{m}) / 3$ is an integer, the nanotube is metallic; otherwise it is a semiconductor [2]. In addition to such unique electrical properties, CNT exhibits extraordinary mechanical properties. The Young's modulus of SWNT has been estimated to be over 1 Tera Pascal and the tensile strength is about $200 \mathrm{GPa}$. The thermal conductivity of CNT has been estimated to be 3000 $\mathrm{W} / \mathrm{mK}$ which is second only to epitaxial diamond. Though CNTs are quite unreactive, they can be functionalized with functional groups either at the tip or on the sidewall of the nanotubes. Given the combination of electronic and mechanical properties and possibilities for functionalization, CNTs offer significant promise for applications in nanodevices, composites, sensors and several other fields. In this article, we present results from our work on chemical vapor deposition (CVD) of nanotubes and show applications of nanotubes as probes in scanning probe microscopy.

\section{CARBON NANOTUBES: AN OVERVIEW}

In this section we provide a concise review of application development to date as well as various growth methods employed to produce both single and multiwalled nanotubes. 


\subsection{CNT Applications}

Since CNT can be metallic or semiconducting depending on chirality, intriguing possibilities arise to put together semiconductor-semiconductor and semiconductor-metal heterojuntions. Initial attempts have focused on the development of a CNT field effect transistor [3, 4]. In this demonstration, one semiconducting single walled nanotube was connected to two metal electrodes and the nanotube was switched from a conducting to an insulating mode by applying a voltage to a gate electrode. CNT is also being explored as a quantum wire $[5,6]$. The theoretical resistance at low bias levels for ballistic conduction is given by $h / 4 \mathrm{e}^{2}$ which is about $6 \mathrm{k} \Omega$. The measured resistance of SWNT and MWNT at present is higher than this theoretical limit due to the contact resistance which is sensitive to contamination and roughness of the contact metal surface. The current carrying capacity of nanotubes is very high as evidenced by the measurements of $10^{7} \mathrm{~A} / \mathrm{cm}^{2}[6]$. CNT is also excellent as a field emitter. The combination of nanosize tips and high conductivity is leading to attempts to develop field emission displays [7]. Carbon nanotube tips have also been explored in atomic force microscope (AFM) based nanolithography [8]. The lithography scheme is field-induced anodization of hydrogen-passivated silicon surface in air which uses a negatively-biased proximal CNT probe tip. The use of CNT tips in semiconductor metrology and surface imaging will be discussed in detail in Section 4 . The ability to functionalize nanotube tips with molecular groups is expected to lead to a breakthrough in chemical and biosensors.

The extraordinary mechanical properties have led researchers to investigate CNTbased composites. The strength-to-weight- ratio of CNT is approximately 600 times greater than for steel. The high thermal conductivity in the axial direction may make CNT useful as a heat sink in integrated circuit chips. Potential also exists to develop body armor, space suits, heat shields, radiators and applications where a high strength-to-weight ratio is an asset. Storage of hydrogen in nanotube bundles has been explored $[9,10]$ but the results to date have been controversial. An initial target of $8 \%$ by weight, ideal for automobile hydrogen batteries, has not yet been met. Storage of lithium has also been attempted for battery applications [11].

\subsection{Nanotube Growth Approaches}

The figure-of-merit for an ideal growth process depends on the application. For nanoelectronics, field emission, displays and sensors, the ability to achieve controlled growth on patterns is important although visionary statements about "electronic device components in a bottle" have been made [12] which would eliminate the need for growth on patterned substrates. For development of composites and other structural applications, the metric would be the ability to produce 'tons a day.' Regardless of the applications and growth technique, the ability to control diameter and chirality of the tubes is critical and this goal has so far been elusive.

An arc process [1] has been used to produce both SWNT and MWNT. This approach involves striking a dc arc discharge in argon between a set of graphite electrodes. The nominal conditions involve $2000-3000 \mathrm{deg}$. C, $100 \mathrm{Amps}$ and 20 volts. This technique produces SWNTs in a mixture of MWNTs and soot. Laser ablation has emerged as an alternative method to produce SWNT [13]. In this process, a target consisting of graphite 
mixed with small amounts of transition metal particles (as catalyst) is placed at the end of a quartz tube enclosed in a furnace. The target is exposed to an argon ion laser beam which vaporizes graphite and nucleates carbon nanotubes in the shock wave just in front of the target. An inert gas, flowing through the reactor and heated to about $1200 \mathrm{dec}$. C by the furnace, carries the vapor and nucleated nanotubes which continue to grow. The nanotubes get deposited on the cooler walls of the tube downstream of the furnace. This method produces about 60-70\% SWNTs with the rest being catalyst particles and graphitic soot. Both of the aforementioned techniques require purification to remove the unwanted impurities and extensive purification methods have been developed [14]. One of the most widely used approaches now is CVD, which involves high temperature dissociation of some form of hydrocarbon feedstock with the aid of a transition metal catalyst [15-17]. In general, nanotubes are not seen without the catalyst. CVD has been very effective for growing MWNTs; however there have not been many reports on SWNTs. CVD has also been effective for growth on patterned substrates [15]. Recently, a plasma source such as a hot filament method [18] or microwave discharge $[19,20]$ has been used to grow MWNTs in arrays as well as films.

\section{CVD OF NANOTUBES}

In this section, we describe our CVD reator, catalyst preparation, results of SWNT and MWNT growth and mechanisms.

\subsection{CVD Reactor}

A typical nanotube growth apparatus consists of a quartz tube enclosed in a furnace. Figure 1 shows a schematic of our CVD reactor which can hold several small substrates. The furnace is capable of isothermal operation within $\pm 1 \mathrm{deg}$. over the entire $0.6 \mathrm{~m}$ length. The growth is carried out at atmospheric pressure with a hydrocarbon feedstock. We use methane for SWNTs and ethylene for MWNTs. Typical growth temperatures are in the range of 700-900 deg. C. A transition metal catalyst is believed to be necessary to promote the growth of nanotubes and a variety of formulations of catalyst materials and techniques to deposit catalyst on the substrates has been suggested in the literature [21]. The quality and quantity of nanotubes depend on the choice of an appropriate combination of the substrate, catalyst and the procedure to apply the catalyst on the substrate. The substrates widely used include silicon, quartz, and alumina. Catalyst application to the substrate is usually achieved by metal evaporation or, sputtering, or through impregnation of liquid based catalysts followed by annealing.

\subsection{Multiwall Carbon Nanotubes}

Our catalyst formulation for multiwall nanotubes consists of mixtures of $\mathrm{Al}, \mathrm{Si}, \mathrm{Fe}$, and $\mathrm{Co}$. A typical preparation of a stock catalyst solution follows. First, $0.5 \mathrm{~g}(0.09 \mathrm{mmol})$ of Pluronic P-123 triblock copolymer was dissolved in $15 \mathrm{~mL}$ of a $2: 1$ mixture containing ethanol and methanol. Next, $\mathrm{SiCl}_{4}(0.85 \mathrm{~mL}, 7.5 \mathrm{mmol})$ was slowly added via syringe into the triblock copolymer/alcohol solution and allowed to stir for $30 \mathrm{~min}$. at room temperature. Stock solutions of $\mathrm{AlCl}_{3} \cdot 6 \mathrm{H}_{2} \mathrm{O}, \mathrm{CoCl}_{2} \cdot 6 \mathrm{H}_{2} \mathrm{O}$, and $\mathrm{Fe}\left(\mathrm{NO}_{3}\right)_{3} \cdot 6 \mathrm{H}_{2} \mathrm{O}$ were prepared at the same concentration of the structure directing agent (SDA) and inorganic salts. The catalyst precursor solutions were filtered through $0.45 \mu \mathrm{m}$ (polytetrafluoroethylene) membranes before deposition. Our substrate consists of $30 \mathrm{~nm}$ iridium sputtered on either quartz or 
silicon. The substate with the ciatalyst formulation was foaded into the furnatce and heated for 700 deg. C for 4 hours in air lo render the callalyst active by the decomposition of the inorganic salts and removal of the SDA.

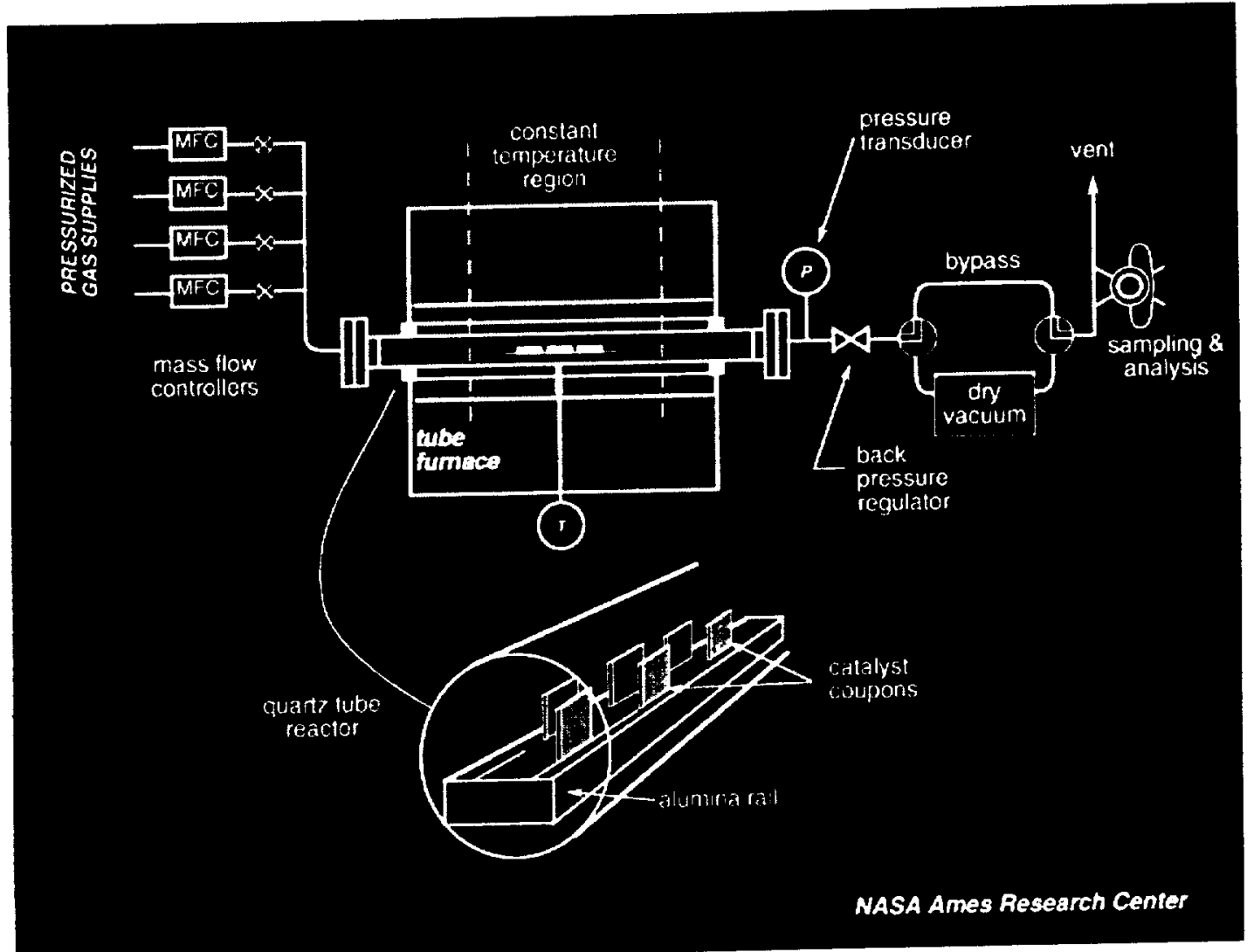

Figure 1. Schematic of the CVD reactor for nanotube grouth

As expected, variations in catalyst stoichiometry. concentration of the SDA. and nature of the substrate led to a wide variety of growth results. The ease of varying the catalyst content in a solution-based preparation described above allowed us to conduct a combinatorial optimization for the evaluation of the catalyst activity of the heterogeneous mixtures [22]. This high throughput technique offers an efficient approach to discover formulations that give researchers control over the nanotuhe type, diameter and length. Figure 2 shows multiwall nanolubes assembled into structures of the order of $100 \mu \mathrm{m}$ in diameter and $300 \mu \mathrm{m}$ tall. In Fig. 2a. the calalyst was assembled into ring-like structures and as a result. the nanotuhes were grown into tall hollow cylinderical structures. In Fig. 2b. the catalyst concentration led to multiwall nanotubes assembled into a tower like structure A close-up view of a tower in Fig. 2c reveals how adjacent nanotubes support each other through Van der Waals interaction in the formation of the towers. Figure 2d is a high resolution TEM image of an individual MWNT and the high quality of the nanotuhe is indicated hy the alignment and general absence of defects along the length of the lube 


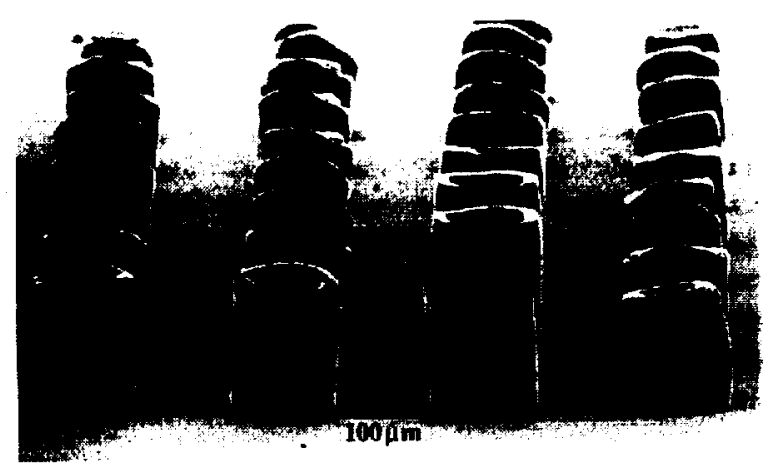

(a)

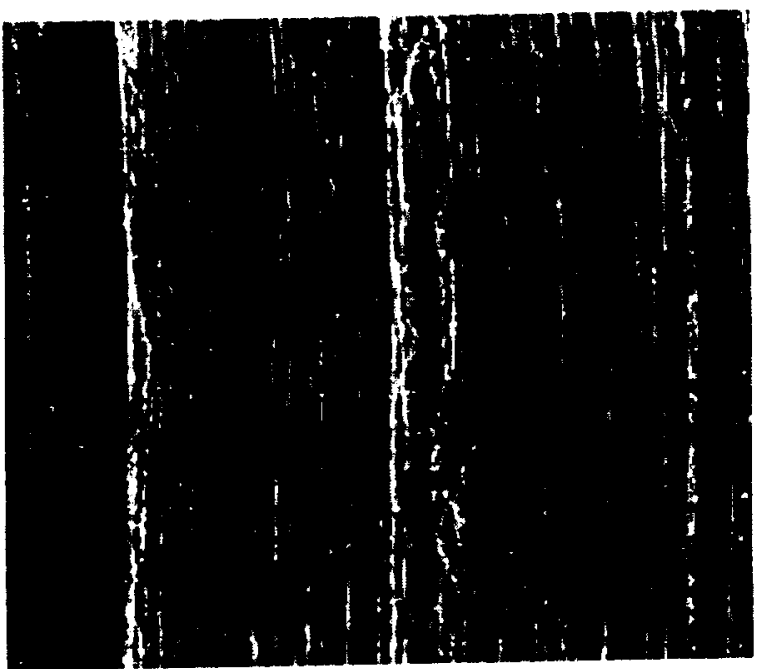

(c)

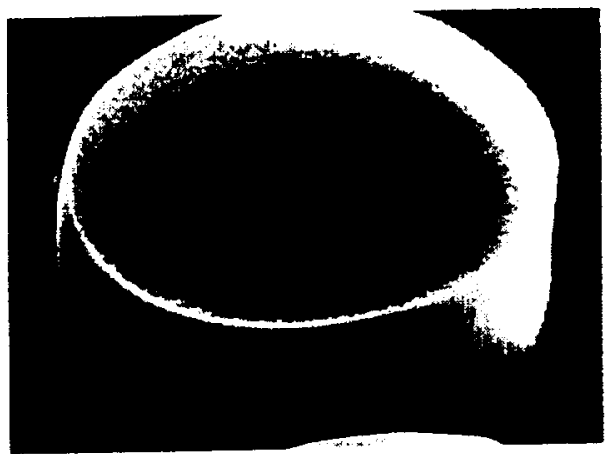

(b)

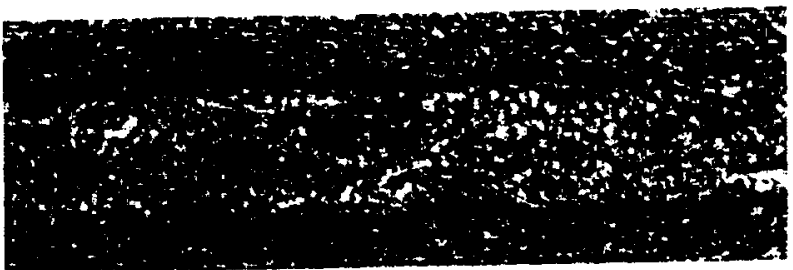

(d)

Figure 2. Multiwall nanotuhes grown by CVD. By changing the concentration of the catalyst solution, nanotubes are assemhled into hollow cylindrical structures (a) or a solid tower (b); (c) high magnification SEM of nanotuhe tower showing individual MWNTs (d) high resolution TEM of an individual MWNT

\subsection{Single Wall Carbon Nanotube}

It is believed that the diameter of the nanotube is controlled by the size of the catalyst particle [23]. In the solution-based catalyst preparation, controlling the particle size below $10 \mathrm{~nm}$ appears to be difficult since particles may agglomerate during annealing. As an alternative, we have pursued catalyst preparation by physical sputtering to enable SWNT growth. This technique uses direct deposition of metal catalysts onto a support surface. A variety of catalyst compositions has been developed which allow for the growth of SWNT with varying surface densities. These formulations incorporate the use of Ir, $\mathrm{Al}, \mathrm{Fe}$, and $\mathrm{Mo}$ by sputtering and produce SWNT densities that range from individual SWNTs on a surface, to thick mats of SWNTs. The mats are composed of ropes of SWNT as shown in Fig. 3. 


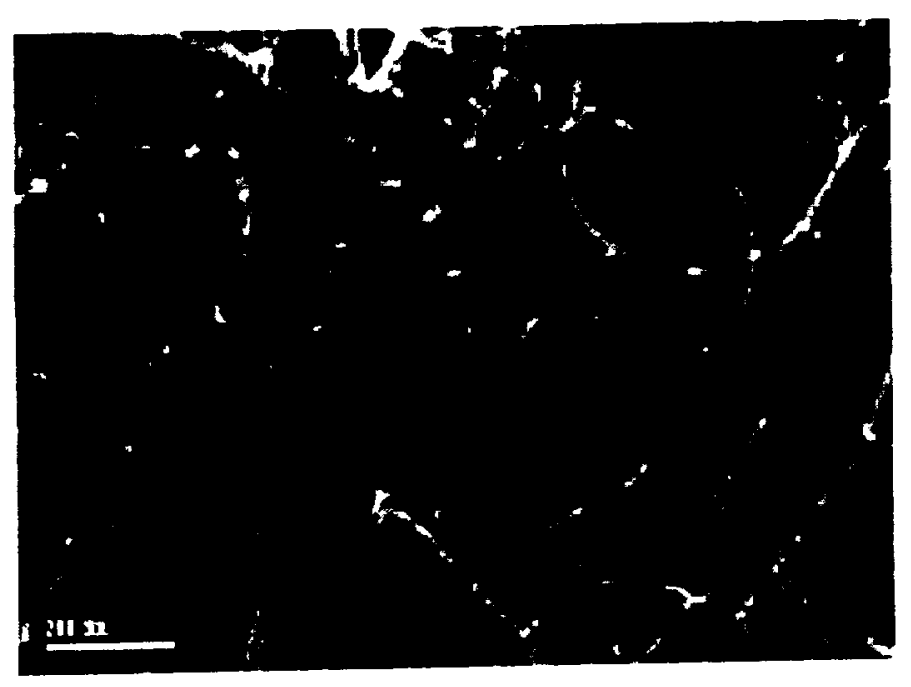

(a)

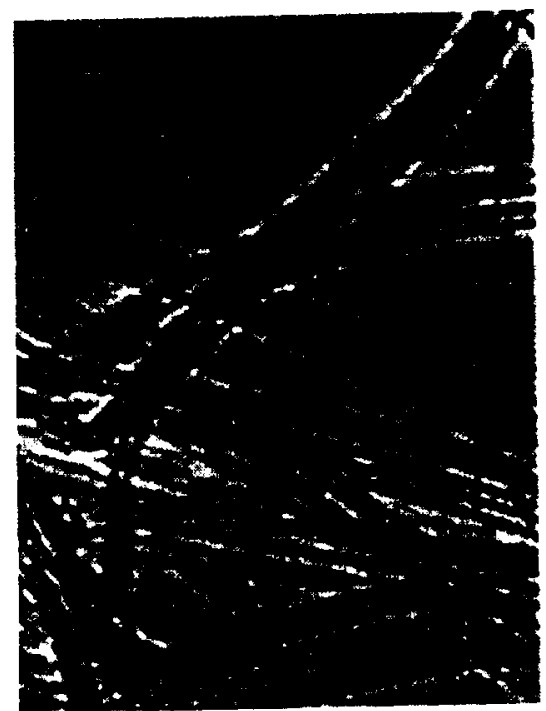

(b)

Figure 3. Ropes of single wall nanotubes by CVD (a) SEM image and (b) high resolution TEM image.

\subsection{Growth Mechanism}

The present understanding of growth mechanism extends from an earlier body of work on the vapor growth of carbon filaments $[24,25]$. Two types of growth modes have been observed by researchers: one in which catalyst particles are rooted at the base of the nanotube and another in which the particles are seen at the tip of the nanotubes. SWNT growth using methane as described in section 3.3 is believed to follow the "base-growth" mechanism [15]. In this case, methane molecules adsorb on the surface of the catalyst particles and dissociate. Subsequently, carbon atoms dissolve and diffuse into the particles. Supersaturation then results in precipitation of carbon in a crystalline tubular form. Ref. 15 argues that when the particles are supported well on a substrate, base growth is the preferable result since the metal species remain pinned to the surface. In contrast, when the metal-substrate interaction is weak, the particle lifts off from the substrate and stays at the end of the tube. This seems to be the case in the gas phase continuous production of nanotubes reported in ref. [16]. Analyzing the results of ref. 16 and other works in the literature, Sinnot et al [23] hypothesize that the formation and growth of nanotubes is an extension of carbon filament growth where the catalyst particle size determines the size of the filament. As the particle size is continuously reduced, a transistion from filament growth to formation of MWNTs and eventually SWNTs is favored. Further experimental evidence through extensive characterization is needed to verify the proposed mechanisms. 


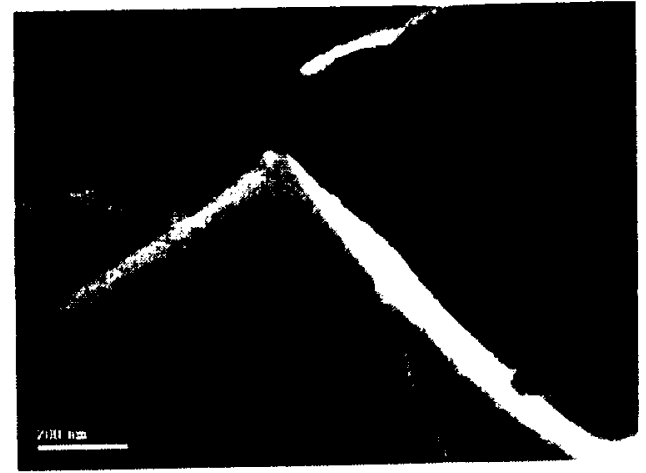

Figure 4. SWNT probe grown by CVD at the pyramidal tip of silicon cantilever.

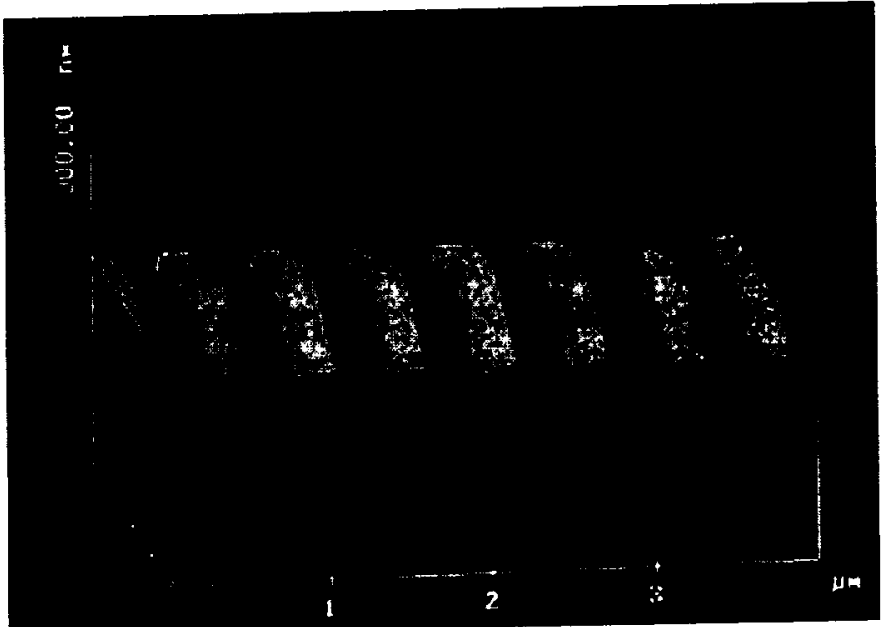

Figure 5. AFM image of line/space photoresist pattern acquired with a MWNT probe.

\section{CNT PROBES IN METROLOGY}

Atomic force microscopy (AFM) has become a widely used metrology tool in semiconductor processing to image and characterize various surfaces. Commercial AFM probes consist of silicon or silicon nitride cantilevers with an integrated pyramidal-shaped tip. These tips now can be made as small as $10 \mathrm{~nm}$ offering reasonable resolution. However, the large cone angle of the order of 30-35 deg. makes it difficult to use these tips for probing narrow and deep features. The biggest drawback of the conventional tip is that it is brittle limiting its use in applications. Alternatively, AFM probes have been prepared by attaching a SWNT or MWNT to the pyramidal silicon tip and shown to offer extraordinary resolution [26]. In addition, the extraordinary strength and the ability to retain structural integrity after deformation made the nanotube probe very robust. However, this pioneering demonstration involved manually attaching a bundle of MWNT to the silicon tip using conducting epoxy, a process which is tedious and not reproducible. Since then, researchers have attempted to develop in situ approaches to attach nanotubes directly to the silicon tip of the AFM cantilever [27]. Of the many possible routes, direct growth by CVD offers perhaps the most practical approach to produce nanotube scanning probes [28]. We have been able to deposit the catalyst at the silicon tip of the cantilever and grow SWNT and MWNT tips directly by CVD. Figure 4 shows a SWNT nanotube probe. Currently. since the growth rate history is not known, we have not been able to control the length of the probe to the desired needs during the growth process. Instead, the probes were shortened afterwards to desired length by applying an electric field. We have been able to routinely use SWNT and MWNT probes prepared by this approach in semiconductor metrology. Figure 5 shows an AFM image, acquired with a MWNT probe, of a line/space pattern of polymeric photoresist ( $300 \mathrm{~nm}$ thickness) on a silicon substrate. This image shows no artifacts due to tip size or shape in contrast to the sloping sides for resist lines normally seen with pyramidal silicon tips. 


\section{CONCLUDING REMARKS}

Carbon nanotubes show tremendous potential for applications in composites, sensors, nanoelectronics and a host of other fields due to their remarkable electronic and mechanical properties. We have shown here that CVD is a versatile technique to produce single and multiwall nanotubes. There are numerous challenges and unanswered questions which need to be addressed to enable large scale production and routine use in applications. Control of diameter and chirality makes the top of the list of challenges. Other issues include understanding of growth mechanisms, ability to grow nanotubes without co-producing other forms of carbon, development of processes for patterned growth as well as integration with processing steps for contact deposition and other necessary functions in sensor and device fabrication, growth of vertically-aligned nanotubes for display and other applications which demand such control, and large scale production techniques for structural applications. These issues are currently receiving worldwide attention.

\section{References}

[1] Iijima S., Nature 354 (1991) 56-58.

[2] Dresselhaus M.S., Dresselhaus G., and Eklund P.C., Science of Fullerenes and Carbon Nanotubes (Academic Press, San Diego, CA, 1996).

[3] Tans S.J., Verschueren A.R.M., and Dekker C., Nature 393 (1998) 49-52.

[4] Martel R., Schmidt T., Shea H.R., Hertel T., and Avouris Ph., Appl. Phys. Lett. 73 (1998) 2447-2449.

[5] Frank S., Poncharal P., Wang Z.L., and de Heer W.A., Science 280 (1998) 17441746.

[6] Avouris Ph., Hertel T., Martel R., Schmidt T., Shea H.R., and Walkup R.E., Appl. Surf. Sci. 141 (1999) 201-209.

[7] Saito Y., Hamaguchi K., Uemura S., Uchida K., Tasaka Y., Ikazaki F., Yumura M., Kasuya A., and Nishina Y., Appl. Phys. A 67 (1998) 95-100.

Dai H., Franklin N., and Han J., Appl. Phys. Lett. 73 (1998) 1508-1510.

[9] Dillon A.C., Jones K.M., Bekkedahl T.A., Kiang C.H., Bethuene D.S., and Heben M.J., Nature 386 (1997) 377-378.

[10] Ye Y., Ahn C.C., Witham C., Fultz B., Liu J., Rinzler A.G., Colbert D., Smith K.A., and Smalley R.E., Appl. Phys. Lett. 74 (1999) 2307-2309.

[11] Zhou O., Private communication.

[12] Smalley R.E., Carbon Nanotubes: Opportunity, Requirements, Challenges, Workshop, Rice University, Houston, TX, 1997.

[13] Guo T., Nikolev P., Thess A., Colbert D., and Smalley R.E., Chem. Phys. Lett. 243 (1995) 49-54.

[14] Bandow S., Rao A.M., Williams K.A., Thess A., Smalley R.E., and Eklund P.C., J. Phys. Chem. 101 (1997) 8839-8842.

[15] Kong J., Soh H.T., Cassell A.M., Quate C.F., and Dai H., Nature 395 (1998) 878881 .

[16] Andrews R., Jacques D., Rao A.M., Derbyshire F., Qian D., Fan X., Dickey E.C., and Chen J., Chem. Phys. Lett. 303 (1999) 467-474.

[17] Cassell A.M., Meyyappan M., and Han J., J. Nanoparticle Res. 2 (2000). 
[18] Chen Y., Wang Z.L., Yen J.S., Johnson D.J., and Prince R.H., Chem. Phys. Lett. 272 (1997) 178-182.

[19] Tsai S.H., Chao C.W., Lee C.L., and Shih H.C., Appl. Phys. Lett. 74 (1999) $3462-$ 3464.

[20] Okai M., Muneyoshi T., Yaguchi T., and Sasaki S., Appl. Phys. Lett. 77 (2000) 3468-3470.

[21] Kind H., Bonard J.M., Forro L., Kern K., Hernadi K., Nillsson L., and Schlapback L., Langmuir 16 (2000) 6877-6883.

[22] Cassell A.M., Verma S., Delzeit L., Meyyappan M., and Han J., Langmuir (2001).

[23] Sinnott S.B., Andrews R., Qian D., Rao A.M., Mao Z., Dickey E.C., and Derbyshire F., Chem. Phys. Lett. 315 (1999) 25-30.

[24] Baker R.T.K., Barber M.A., Harris P.S., Feates F.S., and Waite R.J., J. Catalysis 26 (1972) 51-62.

[25] Baker R.T.K., Harris P.S., Thomas R.B., and Waite R.J., J. Catalysis 30 (1973) 8695.

[26] Dai H., Hafner J.H., Rinzler A.G., Colbert D., and Smalley R.E., Nature 384 (1996) 147-150.

[27] Stevens R.M.D., Frederick N.A., Smith B.L., Morse D.E., Stucky G.D., and Hansma P.K., Nanotechnology 11 (2000) 1-5.

[28] Hofner J.H., Cheung C.L., and Lieber C.M., J. Am. Chem. Soc. 121 (1999) 97509751.

[29] Nguyen C.V., Chao K.J., Stevens R.M.D., Delzeit L., Cassell A.M., Han J., and Meyyappan M., J. Vac. Sci. Tech. (2001). 